\title{
Implementation of a Wireless Temperature Monitoring and Control System for Naval Applications
}

\author{
A. Kroustalis \\ Dept. of Industrial Design \\ and Production \\ Engineering \\ University of West Attica, \\ Athens, Greece
}

\author{
M. Papoutsidakis \\ Dept. of Industrial Design \\ and Production \\ Engineering \\ University of West Attica, \\ Athens, Greece
}

\author{
A. Chatzopoulos \\ Dept. of Industrial Design \\ and Production \\ Engineering \\ University of West Attica, \\ Athens, Greece
}

\author{
D. Piromalis \\ Dept. of Industrial Design \\ and Production \\ Engineering \\ University of West Attica, \\ Athens, Greece
}

\begin{abstract}
The aim of this paper is the presentation of the design and development of an innovative temperature control system according to the mechatronics design philosophy, which be able to integrate into the naval technology. The novelty of this systems is the remotely control and monitoring of any process in a ship relevant to this physical quantity from any point on the ship or even from the shore with a very low cost.
\end{abstract}

\section{Keywords}

ZigBee, WiFi, Wireless Sensors, Wireless control, Wireless monitoring, Mechatronics, Arduino, Naval technology, Temperature, PID Control, Android.

\section{INTRODUCTION}

Nowadays shipping industry is in constant search for solutions to the reduction of the, operating costs and the productivity and efficiency improvement $[1,2,3]$. Through the new era of the 4th industrial revolution there is a trend towards the development of remote effective control and monitoring technologies $[4,5,6]$. Mechatronics integrates the principles of Electrical, Mechanical, Control, Computer, Informatics and Telecommunications engineering to manage complexity, uncertainty and communication on engineered systems $[7,8,9]$.

In modern ships a large number of processes take place which are directly depended on the control of specific physical quantities variables, so the need for innovative control and monitoring systems is essential. According to these, modern mechatronics systems can be the right answer and also to exceed the above needs as they present a number of advantages.

\section{TEMPERATURE MONITORING AND CONTROL IN SHIPS}

Temperature control consists a major importance process as it applies to important procedures directly related with the optimum engines functioning and therefore the seaworthiness of the ship, as well as the appropriate cargo transportation and the ideal crew living conditions.

The key points where temperature control and monitoring is essential are the following $[10,11,12]$ :

- Main engine and electric generators cylinders cooling system.

- Main engine pistons cooling system

- Main engine nozzles cooling system
- Main engine and electric generators oil lubrication cooling system.

- Air downstream of the turbocharger temperature control system.

- Main engine pistons underside supercharging air cooling system.

- Service fuel heating system.

- Accommodation air and water temperature control system.

- Cargo temperature control system.

With the existing technology, temperature control and monitoring is accomplished to specific points on ship including control stations and local panels.

\section{MECHATRONICS AND SHIPPING INDUSTRY}

A modern mechatronic system integrates sensors, actuators, signal conditioning, computer hardware and software with decision and control algorithms, enabling it to manage complexity, uncertainty and communication on engineered systems. A modern mechatronic system is capable of precise control of variables, high performance, efficient power management, low cost, flexibility, reliability, small size, security and interconnectivity $[13,14,15]$.

In this perspective we introduce a system which enables remote control and monitoring by the operator at any point of the ship even locally, with the use of wireless network technologies (Wi-Fi and ZigBee) and low cost embedded systems.

\section{DESIGN AND IMPLEMENTATION OF OUR SYSTEM}

For our project purposes we rely on a typical 2-stroke low speed engine's cylinder cooling system. The general idea of our system design (Fig.1) is constituted of a three way valve which mixes the hot incoming water from the engine with the cold water incoming from the cooler in order to achieve an appropriate water temperature for the cylinder cooling. A sensor monitors the outlet water temperature of the engine and sending a feedback signal to the controller which in turn controls the opening position of the valve. When the water temperature exceeds a predetermined level then an acoustic as a visual indication will be signed (alarm), while at the same time a message will be displayed to the operator. For the local operation of the system a local panel has been developed, where the operator gets the opportunity to select between 
manual or auto mode through a toggle switch, to increase or decrease the desirable temperature set point through 2 pushbuttons in auto mode and to operate the valve in manual mode, opening or closing it through two pushbuttons, to acknowledge an alarm signal through a pushbutton and to set up the temperature level when an alarm signal occurs via a potentiometer. An LCD display has been integrated for the main functions indication.For the remote operation of the system, an android application has been developed which through the web server that the microcontroller supports, the operator gets the ability to enter the desirable temperature set point, to control the valve in a manual way and to acknowledge an alarm signal through virtual buttons, as well as to get all the needed information on the screen of an android based mobile device via a Wi-Fi connection.For the connection between the sensor and the microcontroller we recommend and implement a MESH topology network via the ZigBee protocol which can be expanded to a wider sensor network.

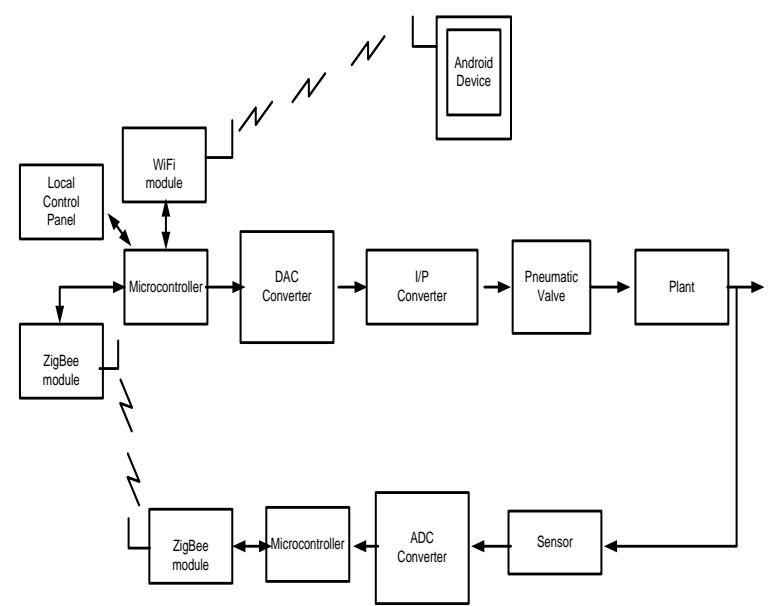

Fig 1: Our system design

\section{HARDWARE DESIGN AND \\ DEVELOPMENT}

In the core of the system we introduce a low cost and opensource, but capable board the Arduino MKR® 1000, which is based on the Atmel ATSAMW25 SoC [16]. The board meets the needs of the system as it provides a powerful 32-Bit low power microcontroller (ARM ${ }^{\circledR}$ Cortex ${ }^{\circledR}-\mathrm{M} 0+$ based) with a clock speed at $48 \mathrm{MHz}, 256 \mathrm{~KB}$ flash memory as well as $32 \mathrm{~KB}$ SRAM, 22 digital and analog I/O which are capable for PWM and are based on ADC and DAC converters, while it provides UART, SPI, I2C and USB serial interface [17]. Also with the embedded SoC (2.4GHz IEEE® 802.11 b/g/n Wi-Fi® ATWINC1500 ) which integrates $802.11 \mathrm{TCP} / \mathrm{IP}$ protocol stack, can provide secure Wi-Fi connectivity. For the appropriate signal output (4-20mA) with which the microcontroller could drive any commercial I/P transducer we developed an electronic circuit which consist of an op-amp which drives two amplifiers transistors, where the output signal from the microcontroller is been amplified and with the use of two resistors a current loop is implemented.

For the monitoring of the outlet water temperature we introduce the 1-Wire interface digital sensor DS18B20 [18], with a unique 64-bit serial number, which provide 9-bit to 12bit temperature measurements in the range of $-550 \mathrm{C}$ to $+1250 \mathrm{C}$ with an accuracy of $\pm 0.50 \mathrm{C}$ from $-100 \mathrm{C}$ to $+850 \mathrm{C}$.

For the implementation of the ZigBee mesh sensor network we introduce two ZigBee compatible XBee® XB24-Z7WIT-
004 [19] modules with the embedded KH XBee ZigBee mesh firmware, which can provide a data transfer rate up to $250 \mathrm{Kbps}$, at a range up to $120 \mathrm{~m}$. For the interconnection of the sensor and the ZigBee module through uart interface we introduce a less powerful board, the arduino UNO Rev $3 \AA$ board [20] based on an 8-bit microcontroller with 32KB flash memory, as 2 KB SRAM, 14 digital Inputs/Outputs pins, of which 6 provide PWM output, and 6 analog inputs pins.For the information display on the local panel we introduce a $20 \times 4$ character LCD display with an embedded I2C interface controller.

\section{SOFTWARE DESIGN AND DEVELOPMENT}

The development of the necessary code for the microcontrollers was made using the Arduino Integrated Development Environment (IDE), The code for the MKR1000® board is consisting of three main sections, the variable declaration section, the setup section and the loop section. At the first section we insert 5 libraries which allow the I2C and SPI interface development, the LCD display function through an $\mathrm{I} 2 \mathrm{C}$ protocol, network control and the PID control.

At the setup section initialization and start up operations are performed, regarding the input/ output pins will be used, the PID control, the LCD display, the serial interface, and the network control.

At the void loop section the main code is imported in a repeatedly loop until the board is powered off or is reset. In here a set of instructions is inserted regarding the operating support of the web server, the data processing from the inputs signals of the system and the rendering of output signals to it. Specifically the key points of the algorithm are that the web server and therefore the microcontroller, is programmed to response at specific http requests received from the android application via WiFi connection, while a $5 \mathrm{sec}$ page refresh is set. Through the PID algorithm the data from the sensor in the form of a byte is been processing, rendering an analog output to the system. With the specific PID library a set with two different values at the tuning parameters can be implemented so the controller behavior be more conservative when the process value is near set point and more aggressive when it is farther away.For the UNO Rev $3 \AA$ board we introduce 2 libraries, for the 1-Wire interface development and the sensor's interconnection development.

\subsection{XBee® modules configuration}

The XBee ${ }^{\circledR}$ modules configured via the XCTU platform, where the first module configured as the coordinator of the MESH network and the second as a router while there is the possibility of expanding the network.

\subsection{Android application development}

The Android application was developed with the MIT App Inventor. For the operator interface environment we introduce 6 virtual buttons with which the operator can enter the set point of the desired temperature, to handle the valve, as well as to acknowledge the alarm signal and to display history events on a text screen. Also a web viewer monitoring the web server of the microcontroller. A continuing running loop is responsible for real time monitoring of alarm messages and gives a sound signal in combination with vibration when an alarm signal occurred via the mobile device. 


\section{DISCUSSION/ CONCLUSION}

Controlling and monitoring the temperature variable as mentioned above is a major issue in naval technology. This study will fill a gap in existing systems, both in terms of cost and functionality. The implementation of our system was carried out by already low - cost materials in a form of singleboard microcontrollers, with the positional to a further cost reduction, through the use of individual raw materials and mass production. Wireless data transfer means reducing the large amount of cables used up to now. In addition with the compressed installation time this can lead to an additional reduction in the installation cost of the system, while it consist a suitable retrofit solution it is possible to co-operate with elements and technologies that are probably already on a ship. The possibility of wireless control and monitoring by the operator offer advantages in terms of safety and working routine for the crew as for the facilities. The operator will be able to monitor and control the system in real time anywhere on the ship there is network coverage and may react immediately in a malfunction event.

As a scalable and flexible system can meet the required needs of a plant while respectively new functions can be easily added as well as modified and extended in any case. Especially, the microcontroller's control and supervision over a local network can be extended and carried out over the internet through the existing satellite communication system provided by a ship.Another important application of the system would be its integration to the refrigerated intermodal containers, where it could be possible the real time temperature or more variables monitoring and control from the ship or the land.At this stage, in order to adjust the parameters $\mathrm{Kp}, \mathrm{Ki}$ and $\mathrm{Kd}$ for the PID control, should be used one of the known methods according to Ziegler-Nichols or Cohen-Coon. In a future study we propose to be introduced a library which allows the auto-tuning of these parameters. Also in a further study we suggest to study other techniques of microcontrollers programming and to conduct conclusions about the system performance in relation to them.

Most commercial ships today have a local Wi-Fi or Ethernet or combination of both technologies network with Wi-Fi network range, limited to the superstructure of the vessel. Under these conditions, wireless routers should be deployed at strategic locations in the engine room, depending on the installation, to achieve a wireless link in this difficult environment. According to these, a study should be carried out in a real engine room environment in order to draw conclusions before the installation.In the end, in a future study, the regulations regarding the measuring and control instruments of the ships must been reviewed and compared with the features of our system in order to make the necessary modifications and align with them.

\section{ACKNOWLEDGMENTS}

All authors would like to thank the University of West Attica and specifically the Post Graduate Program of Studies (MSc) "New Technologies in Shipping and Transport", for the financial support provided to them to undertake this research project.

\section{REFERENCES}

[1] Lai, K-H., Lun, V., Wong, C. \& Cheng, T.. (2011). Green shipping practices in the shipping industry: Conceptualization, adoption, and implications. Journal of Resources, Conservation and Recycling, 55, 631-638.
[2] UNCTAD, 2017. Review of Maritime Transport 2017, UNCTAD/RMT/2017. New York: United Nations.

[3] UNCTAD, 2016. Review of Maritime Transport 2016, UNCTAD/RMT/2016. New York: United Nations.

[4] Kobylinski, L.. (2016). Marine Transport and the Fourth Industrial Revolution. In journal of Transport, 111, pp. 270-278.

[5] Levander, O. (2017), Forget Autonomous CarsAutonomous Ships Are Almost Here. https://spectrum.ieee.org/transportation/marine/forgetautonomous-cars-autonomous-ships-are-almost-here $(17 / 01 / 2018)$

[6] 12MESA - Maritime Europe Strategy Action Web Site. https://www.waterborne.eu/vision/ (17/01/2018)

[7] Auslander, D. \& Kempf, C. (1996). Mechatronics: Mechanical System Interfacing. New Jersey: Prentice Hall.

[8] Harshama, F., Tomizuka, M., \& Fukuda, T.. (1996). Mechatronics - "What is it, Why and How?" An editorial. Journal of IEEE/ASME Transactions on Mechatronics, 1 pp. 1-4.

[9] Tomizuka, M. (2001). Mechatronics: from the 20th to 21st Century. Journal of Control Engineering Practice, $10,877-886$.

[10] McGeorge, D., H.. (2002). Marine Auxiliary Machinery. 7th Ed. Oxford: Elsevier Science Ltd.

[11] Taylor, A., D.. (2003). Introduction to Marine Engineering. Burlington: Elsevier Ltd.

[12] Woodyard, D.. (2009). Marine Diesel Engines and Gas Turbines. 9th Ed. Oxford: Elsevier Ltd.

[13] Auslander, D. \& Kempf, C. (1996). Mechatronics: Mechanical System Interfacing. New Jersey: Prentice Hall.

[14] Bishop, H., R.. (2006). Mechatronics - An Introduction. Texas: CRC Press.

[15] Bradley, D.. (2004). What is mechatronics and why teach it? International Journal of Electrical Engineering Education, 41, pp. 275-291.

[16] ATSAMW25-MR210PB Atmel Datasheet. IEEE 802.11 $\mathrm{b} / \mathrm{g} / \mathrm{n}$ SmartConnect Wi-Fi Module Datasheet. http://ww1.microchip.com/downloads/en/DeviceDoc/At mel-42618-SmartConnect-ATSAMW25MR210PB_Datasheet.pdf (20/01/2018)

[17] 13MKR 1000, Genuino Board Technical Specifications and Documentation. https://store.arduino.cc/arduinomkr1000 (20/01/2018)

[18] DS18B20 Datasheet. Programmable Resolution 1- Wire Digital Thermometer. Maxim Integrated.

[19] XBee® ${ }^{\circledR}$ /XBee - Pro S2C Zigbee ${ }^{\circledR}$ RF Module Datasheet.https://www.digi.com/resources/documentatio n/digidocs/pdfs/90002002.pdf (20/01/2018)

[20] UNO Rev3, Arduino Board Technical Specifications and Documentation.https://store.arduino.cc/arduino-uno-rev3 $(20 / 01 / 2018)$ 